\title{
Sus1 is recruited to coding regions and functions during transcription elongation in association with SAGA and TREX2
}

\author{
Pau Pascual-García, ${ }^{1,4}$ Chhabi K. Govind, ${ }^{2,4}$ Ethel Queralt, ${ }^{1,5}$ Bernardo Cuenca-Bono, ${ }^{1}$ Ana Llopis, ${ }^{1}$ \\ Sebastián Chavez, ${ }^{3}$ Alan G. Hinnebusch, ${ }^{2}$ and Susana Rodríguez-Navarro ${ }^{1,6}$ \\ ${ }^{1}$ Centro de Investigación Príncipe Felipe (CIPF), E-46012 Valencia, Spain; ${ }^{2}$ Laboratory of Gene Regulation and Development, \\ National Institute of Child Health and Development, National Institutes of Health, Bethesda, Maryland 20892, USA; \\ ${ }^{3}$ Departamento de Genética, Universidad de Sevilla, E-41012 Seville, Spain
}

\begin{abstract}
Gene transcription, RNA biogenesis, and mRNA transport constitute a complicated process essential for all eukaryotic cells. The transcription/export factor Sus1 plays a key role in coupling transcription activation with mRNA export, and it resides in both the SAGA and TREX2 complexes. Moreover, Sus1 is responsible for GAL1 gene gating at the nuclear periphery, which is important for its transcriptional status. Here, we show that Sus1 is required during transcription elongation and is associated with the elongating form of RNA Polymerase II (RNAP II) phosphorylated on Ser5 and Ser2 of the C-terminal domain (CTD). In addition, Sus1 copurifies with the essential mRNA export factors Yra1 and Mex67, which bind to the mRNA cotranscriptionally. Consistently, ChIP analysis reveals that Sus1 is present at coding regions dependent on transcription in a manner stimulated by Kin28-dependent CTD phosphorylation. Strikingly, eliminating the TREX2 component Sac3 or the SAGA subunit Ubp8 partially impairs Sus1 targeting to coding sequences and upstream activating sequences (UAS). We found, unexpectedly, that Sgf73 is necessary for association of Sus1 with both SAGA and TREX2, and that its absence dramatically reduces Sus1 occupancy of UAS and ORF sequences. Our results reveal that Sus1 plays a key role in coordinating gene transcription and mRNA export by working at the interface between the SAGA and TREX2 complexes during transcription elongation.
\end{abstract}

[Keywords: Sus1; SAGA; TREX2; transcription elongation; mRNA export]

Supplemental material is available at http://www.genesdev.org.

Received April 14, 2008; revised version accepted August 21, 2008.

Gene expression in eukaryotes depends on the coordinated action of several multiprotein complexes. These complexes regulate transcription, mRNA biogenesis, and the export of a mature mRNA out of the nucleus (Komili and Silver 2008). The interplay between these factors is necessary to ensure that a correct message will be translated. Work from many laboratories during the last few years has uncovered coupling mechanisms between the different machineries involved in decoding the DNA. The TREX1 protein complex (Sub2, Yra1, and THO components) (Strasser et al. 2002) constitutes one of the first examples of integrating different steps during mRNA biogenesis and nuclear export. THO subunits of TREX1 are able to interact with chromatin, whereas Sub2 and Yral contact the mRNA and promote the re-

\footnotetext{
${ }^{4}$ These authors contributed equally to this work.

${ }^{5}$ Present address: Cancer Epigenetics and Biology Program (PEBC), Institut Català d'Oncologia, Av. Gran Via S/N, 08907 L'Hospitalet de Llobregat, Barcelona E-08907, Spain.

${ }^{6}$ Corresponding author.

E-MAIL srodriguez@cipf.es; FAX 34-96-328-9701.

Article is online at http://www.genesdev.org/cgi/doi/10.1101/gad.483308.
}

cruitment of mRNA-binding proteins during elongation (Abruzzi et al. 2004). A further link between transcription and mRNA export was revealed by the identification of Sus1 (Rodríguez-Navarro et al. 2004). We found that this small protein interacts with the transcriptional coactivator SAGA and the nuclear pore-associated complex composed of Sac3-Thp1-Cdc31 (recently termed TREX2) (Kohler and Hurt 2007). We demonstrated that its role in mRNA export is likely carried out through physical interactions with TREX2, via Sac3-CID (Cdc31-interacting domain) (Fischer et al. 2004). In addition, we showed that GAL1 gene tethering to the nuclear periphery depends on Sus1 (Cabal et al. 2006). Sus1 function is required for accurate chromatin positioning in the nucleus, and, therefore, it influences the transcriptional status of a gene. In this context, recently it has been shown that Sus1p, Sac3p, and Thp1p mediate the post-transcriptional tethering of active genes to both the nuclear rim and the nonnascent mRNP (Chekanova et al. 2008).

Besides its clear involvement in gene gating and mRNA transport, Sus1 is a component of the evolution- 
arily conserved SAGA coactivator complex (STAGA/ TFTC in higher eukaryotes). SAGA is organized into modules with distinct functions in the transcription process (Baker and Grant 2007). The SAGA complex is recruited by activators to promoter upstream activation sequences (UASs), where it facilitates access of general transcription factors (GTFs) to chromatin (Cosma et al. 1999; Bhaumik and Green 2001; Larschan and Winston 2001; Swanson et al. 2003). SAGA contains two enzymatic activities involved in post-translational histone modifications. Histone acetylation is carried out by the SAGA subunit Gen5 (Candau et al. 1997; Grant et al. 1997), whereas the ubiquitin protease Ubp8 is necessary for histone deubiquitinylation (Henry et al. 2003). SAGA-dependent histone modifications play a crucial role in the regulation of different steps during gene expression (for review, see Weake and Workman 2008). We and others have shown that Ubp8, together with Sus1 and Sgf11, form a distinct functional module in SAGA that is required for the deubiquitinylation of $\mathrm{H} 2 \mathrm{~B}$ (Ingvarsdottir et al. 2005; Lee et al. 2005; Kohler et al. 2006). Our work showed that Sus1p forms a stable subcomplex with Sgf11p and Ubp8p and plays a role in both histone $\mathrm{H} 2 \mathrm{~B}$ deubiquitinylation and the maintenance of steadystate H3 methylation levels (Kohler et al. 2006). Binding of Sus1 to SAGA depends on the deubiquitinylating enzymes Ubp8 and Sgf11. Thus, the deubiquitinylation module could work at the junction between SAGA-dependent transcription and nuclear mRNA export. Apart from the established role of SAGA in transcription activation, two recent studies suggest that SAGA also localizes at the coding sequences, reinforcing the previously proposed role for the complex in elongation (Desmoucelles et al. 2002). In fact, Gcn5-dependent acetylation promotes nucleosome eviction and appears to enhance processivity of RNA Polymerase II (RNAP II) during transcription elongation (Govind et al. 2007). The association of SAGA with coding sequences is dependent on phosphorylation of the C-terminal domain (CTD) of RNAP II subunit Rpb1, indicating that SAGA might interact with actively transcribing RNAP II during elongation. Moreover, new findings reveal a mechanism by which H2B ubiquitinylation acts as a barrier for the association of Ctklp with the coding regions of active genes, while subsequent deubiquitinylation by Ubp8p triggers Ctk1p recruitment, suggesting an overall role for SAGA in regulating the entire transcriptional process (Wyce et al. 2007).

Several recent studies have shown that Sus 1 function is conserved in evolution. As revealed for yeast, Drosophila Sus $1 / \mathrm{E} / \mathrm{y}) 2$ is a subunit of the SAGA/TFTC-type histone acetyltransferase complex, and it concentrates at the nuclear periphery (Kurshakova et al. 2007b). E(y)2 interacts with the nuclear pore complex (NPC) in a complex with X-linked male sterile 2 (Xmas-2, a putative ySac3 ortholog) to regulate mRNA transport. Drosophila Sus1 functions in the anchoring of a subset of transcription sites to the NPCs to achieve efficient transcription and mRNA export. In addition, it has been shown that $\mathrm{E}(\mathrm{y}) 2 / \mathrm{Sus} 1$ is essential for the barrier activity of $\mathrm{Su}(\mathrm{Hw})$ - dependent insulators in Drosophila (Kurshakova et al. 2007a). Recently, Zhao et al. (2008) verified that Sus1, Ubp8, and Sgf11 are conserved in humans (ENY2, USP22, and ATXN7L3, respectively) and that they form the deubiquitinylation module in human STAGA. Furthermore, they show for the first time that ENY2, USP22, and ATXN7L3 are required as cofactors for the full transcriptional activity of nuclear receptors (Zhao et al. 2008).

All these findings in yeast, Drosophila, and human cells suggest that Sus1 may provide a physical link between promoter-bound activators and the mRNA export machinery. However, the mechanism by which Sus1 functions in these processes remains unclear. In this study, we investigated mechanisms underlying Sus1's role in coupling transcription and export. We show that Sus 1 is required for gene transcription in a length-dependent way, which suggests an active role in mRNA biogenesis during transcription elongation. Consistent with this, we found that Sus1 copurifies with the elongating form of RNAP II phosphorylated on Ser5 and Ser2 of the CTD, the mRNA adaptor Yra1, and the export factor Mex67. We also provide evidence that Sus1 is associated at high levels with the $A R G 1$ gene coding sequence and that Sus1 occupancy of the ORF requires transcription and Ser5 phosphorylation of the CTD. Our findings demonstrate that loss of the SAGA subunit Sgf73 prevents Sus1 binding to SAGA and, unexpectedly, partially disrupts Sus1-TREX2 association. Whereas Ubp8 and Sac3 both promote association of Sus1 with the ARG1 UAS and coding region, Sgf73 is more critically required for these interactions. Our data suggest a mechanism by which Sus1 plays a pivotal role in transcription during elongation mediated by both the SAGA and TREX2 complexes.

\section{Results}

Sus1 is able to activate transcription and is necessary for $m R N A$ biogenesis during transcription elongation

One of the best-characterized functions of the SAGA complex is its role in transcription activation, mainly due to its ability to modify histones in chromatin (for reviews, see Baker and Grant 2007; Nagy and Tora 2007). Since Sus1 is part of the SAGA complex, we tested whether Sus1 is able to activate transcription when fused to the bacterial LexA DNA-binding domain. CTY strains containing a lexAop-lacZ reporter were transformed with a plasmid encoding a LexA-Sus1 fusion (pBTM-Sus1) or empty vector (pBTM-Empty), and $\beta$-galactosidase activity was measured in exponentially growing cultures of the resulting transformants. We observed $\sim 130$-fold higher activity in transformants harboring pBTM-Sus1 compared with those containing empty vector (Fig. 1A). Our results clearly show that Sus 1 is able to activate transcription. To determine whether activation by LexA-Sus1 requires the SAGA complex, we measured $\beta$-galactosidase activity in three different SAGA mutants that impair either Sus1-SAGA association $(s g f 11 \Delta)$, SAGA HAT activity $(g c n 5 \Delta)$, or PIC-assem- 
A

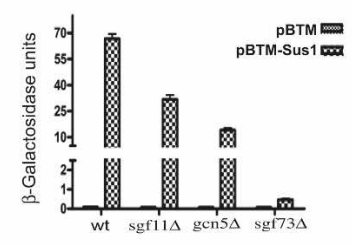

C

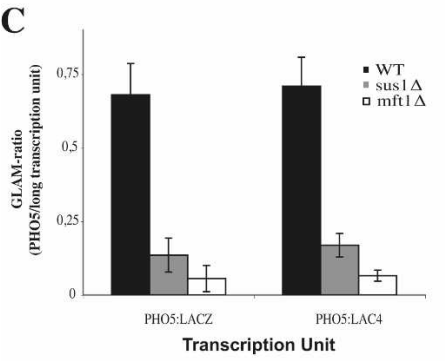

$\mathbf{E}$

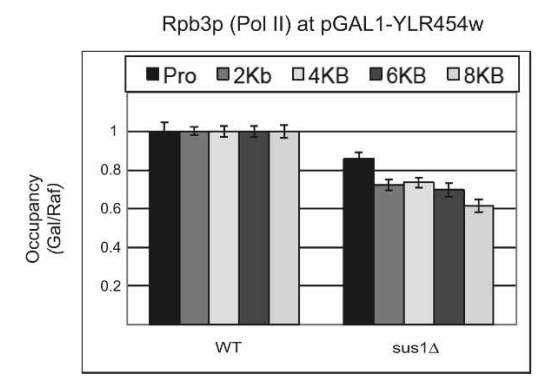

Figure 1. Sus1 activates transcription and is important for gene transcription in a gene-length-dependent manner. $(A)$ Wild-type (CTY) and the isogenic SAGA mutants sgf11s, gcn5s, and $s g f 73 \Delta$ cells were transformed with pBTM-empty or pBTM-Sus1 plasmids. Transformants were grown to mid-logarithmic phase in selective SC-TRP medium. $\beta$-galactosidase activity was assayed in cells and Miller units are represented. Values are mean $\beta$-galactosidase activities from four transformants (error bars represent SE). (B) Different lengths of transcription units used in this work for the GLAM-ratio assays. (C) Wild type (BY4741) and isogenic sus $1 \Delta$ and $m f t 1 \Delta$ were transformed with the centromeric plasmids described in B. GLAM-ratios are calculated as the relative levels of acidic phosphatase activity expressed by the indicated transcription unit $\left(P_{G A L^{-}}\right.$PHO5 LacZ [2] or $P_{G A L^{-}}$ PHO5 LAC4 [3]), with respect to the acid phosphatase activity from the shortest transcription unit $\left(P_{G A L}-P H O 5\right.$ [1]). For each strain, the average of at least three experiments was considered (error bars represent SE). (D) qRT-PCR showing the expression levels of PHO5 from the three transcription units (T. unit) described in $B$ for wild-type and sus $1 \Delta$ cells. Amplification of SCR1 was used as a loading control. (E) Comparison of Pol II (Rpb3p) occupancy at different locations through the $P_{G A L 1^{-}}$ YLR454W gene following galactose induction in wild-type and sus $1 \Delta$ cells, expressed as the ratio of occupancies in galactose versus rafinose medium. All error bars represent the SEM.

bly $(s g f 73 \Delta)$. As shown in Figure 1A, $\beta$-galactosidase activity derived from lexA-Sus1 activation is reduced in all three mutants, but to different extents. The $s g f 73 \Delta$ strain shows the most severe reduction, with levels similar to the background (0.15 units), whereas activation is reduced by a factor of seven for gcn5s. Interestingly, in $s g f 11 \Delta$ the reduction is less severe, which suggests that in this context, binding of lexA-Sus1 to SAGA is not completely eliminated. In conclusion, Sus1 activates transcription dependent on functional SAGA.

Recent work indicates an active role for SAGA during transcription elongation (Govind et al. 2007; Wyce et al. 2007). The fact that Sus1 is part of SAGA and is able to activate transcription prompted us to test whether Sus1 participates in elongation. To this end, we measured the efficiency of gene-length-dependent mRNA accumulation using the GLAM-ratio assay, (Morillo-Huesca et al. 2006). Wild-type and sus1s strains were transformed with three plasmids harboring transcription units of different length containing the same PHO5 coding sequences but variable 3 ' untranslated sequences, all under the same (GAL1) promoter. The ratios of acid phosphatase activity for the short transcript versus the long transcripts (GLAM-ratios) were measured for each strain (Fig. 1B). The THO complex mutant mft1s, known to be defective in transcription elongation and recombination (Chavez et al. 2000), was examined in parallel. As presented in Figure 1C, the GLAM-ratios for sus1D were significantly reduced from wild-type levels, nearly to the extent observed for $m f t 1 \Delta$. The gene-length-dependent defect obtained for sus $1 \Delta$ was further confirmed by quantitative RT-PCR (qRT-PCR) assays performed with cDNAs generated from wild-type and sus $1 \Delta$ transformants bearing the different transcription units. PHO5 and $S C R 1$, as a loading control, were amplified and compared in an ethidium bromide-stained gel (Fig. 1D). Our results show that PHO5 expression is decreased in sus $1 \Delta$ when expressed from long transcription units containing either lacZ or LAC4 3' untranslated sequences, but is not affected when PHO5 is transcribed from the short transcription unit. These results are compatible with a role of Sus1 during transcription elongation. To rule out that the observed defect in sus $1 \Delta$ is due to an mRNA export defect, we also analyzed the GLAM-ratios for the nucleoporin mutant nup2s. Mutant cells lacking Nup2 do not exhibit a significant decrease in GLAM-ratios (Supplemental Fig. 1). To analyze the role of SUS1 in transcription elongation, we examined RNAP II occupancy across the 8-kb YLR454W ORF placed under the control of the GAL1 promoter, under galactose-inducing conditions. As shown in Figure 1E, the sus1D mutant showed reduced RNAP II occupancy across the $P_{\text {GAL1 }}$-YLR454W gene compared with wild-type cells. In addition, a small reduction in Pol II at the promoter was also observed. Together, these results indicate a role for SUS1 in transcription elongation and suggest that the phenotype is not an indirect consequence of an mRNA export block.

Sus1 interacts with hyperphosphorylated forms of RNAP II and the mRNA export factors Yra1 and $M e x 67$

Our published results favor a model in which Sus1 couples transcription activation and export by physical interactions with the SAGA complex and the NPCassociated mRNA export complex Sac3-Thp1-Cdc31 
(TREX2) (Fischer et al. 2004; Rodríguez-Navarro et al. 2004). Nevertheless, how this coupling takes place remains unclear. The strong gene-length-dependent transcription defect we observed for sus $1 \Delta$ could reflect an active role of Sus1 in mRNA biogenesis. To gain insight into the mechanisms underlying Sus1-dependent transcription, we analyzed whether Sus1 interacts with factors involved in different steps during mRNA biogenesis. TAP-tagged Sus1 and Ada2, as a bona fide SAGA component, were affinity purified, and the calmodulin eluates were checked for the presence of different proteins by Western blot analysis. The results clearly demonstrate that Sus1 copurifies with RNAP II (Fig. 2A). Given that phosphorylation of RNAP II-CTD Ser5 and Ser2 residues constitutes a signal for promoter clearance and effective elongation (for review, see Saunders et al. 2006), we tested the presence of these RNAP II forms in a Sus1TAP purification. As shown in Figure 2A, Sus1 copuri-

\section{A}
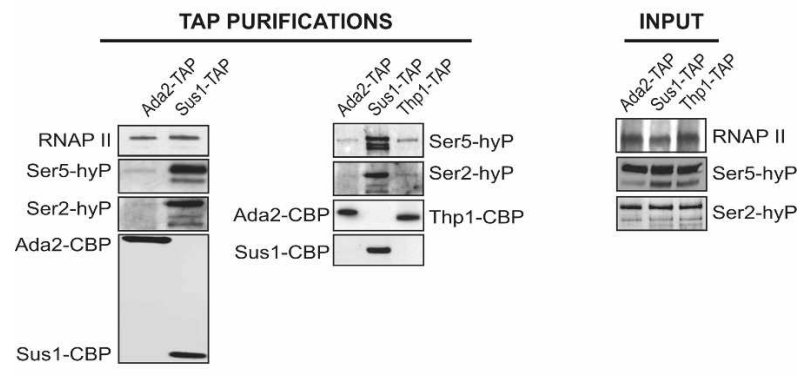

B

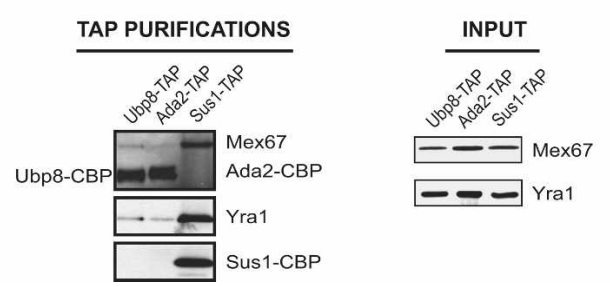

Figure 2. Sus1 copurifies with RNAP II and the mRNA export factors Yra1 and Mex67. (A) TAP purification of Ada2, Thp1, and Sus 1 TAP-tagged cells was performed, and the eluates were resolved by $4 \%-12 \%$ gradient gels, transferred, and immunoblotted. The presence of RNAP II, Ser5-hyP, and Ser2-hyP was revealed by the use of the appropriated antibodies. Equal loading of each TAP purification was ensured by detecting the bait Ada2-CBP or Sus1-CBP with an anti-CBP antibody. Input level of each protein before the TAP purification was detected from the lysates by Western blot using the mentioned antibodies. (B) TAP purification of Ubp8, Ada2, or Sus1 TAP-tagged cells was performed and the eluates were used for Western blot analysis as described in $A$. The presence of Yral and Mex67 was revealed by the use of the appropriate antibodies. Equal loading was ensured by detecting the baits Ubp8-CBP, Ada2-CBP, or Sus1-CBP with an anti-CBP antibody. Input level of each protein before the TAP purification was detected from the lysates by Western blot using the mentioned antibodies. fies with RNAP II hyperphosphorylated in both residues, Ser5 (Ser5-hyP) and Ser2 (Ser2-hyP). Interestingly, association of Ser5-hyP and Ser2-hyP RNAP II with Sus1 likely occurs via dynamic interactions during transcription elongation, since SAGA (Ada2-TAP) or TREX2 (Thp1-TAP) subunits didn't substantially enrich these RNAP II forms when purified (Fig. 2A, middle panel). Therefore, we conclude that Susl mainly interacts with the Ser5 and Ser2 hyperphosphorylated forms of RNAP II, which are indicative of promoter clearance and effective transcription elongation.

Several studies have established links between factors involved in transcription and mRNA export (for review, see Cole and Scarcelli 2006). Since Sus1 interacts dynamically with the transcription apparatus, it is conceivable that other proteins involved in mRNA biogenesis and export also bind to Sus 1 during gene expression. To identify new dynamic interactions, we studied whether Sus 1 copurifies with the essential mRNA export factors Yra1 and Mex67. As shown in Figure 2B, Sus1 associates with both Yral and Mex67. In contrast, strong interactions with Yral and Mex67 were not observed when Ada2-TAP or Ubp8-TAP was used as bait for the purification, despite comparable levels of Yral and Mex67 in the starting cell extracts (Fig. 2B). Therefore, we conclude that Sus 1 physically interacts with the key mRNA export factors Yral and Mex67, suggesting a more general role for Sus1 in mRNA biogenesis.

Sus1 is targeted to coding regions in a manner stimulated by CTD Ser5 phosphorylation by Kin28

The results presented so far in this study strongly suggest that Sus 1 could have an active role during elongation in coding sequences and that this function could be mediated by SAGA. In fact, as described in the introduction, several SAGA subunits have been shown to be present at coding regions. To analyze whether Sus1 is associated with RNAP II-transcribed regions, we studied Sus1 occupancy to the SAGA-regulated gene ARG1. Previously, we showed by chromatin immunoprecipitation (ChIP) analysis that SAGA subunits are recruited to the UAS and coding region of $A R G 1$ by the activator Gen $4 p$ in amino-acid-limited cells where Gen 4 p binding to UAS elements is induced (Govind et al. 2007). Similarly, we observed high-level association of Myc-Sus1 with the UAS and the $5^{\prime}$ and $3^{\prime}$ ends of the ARG1 ORF in wildtype cells starved for Ile and Val with the anti-metabolite sulfometuron (SM) (Fig. 3A,B; data not shown). In gcn $4 \Delta$ cells, in contrast, nearly background levels of Myc-Sus1 were detected at the UAS and ORF. These data demonstrate that Sus1 is targeted by Gen $4 p$ to the UAS and coding regions during transcriptional induction of ARG1. To extend the analysis of Sus1 presence at the coding region of a noninducible gene, we also investigated Sus1 association with the $A D H 1$ gene. We found that Sus1 is also bound to both promoter and coding region of the housekeeping gene $A D H 1$ (Supplemental Fig. 2A).

Given that Sus1 physically interacts with the Ser5-hyP 
A
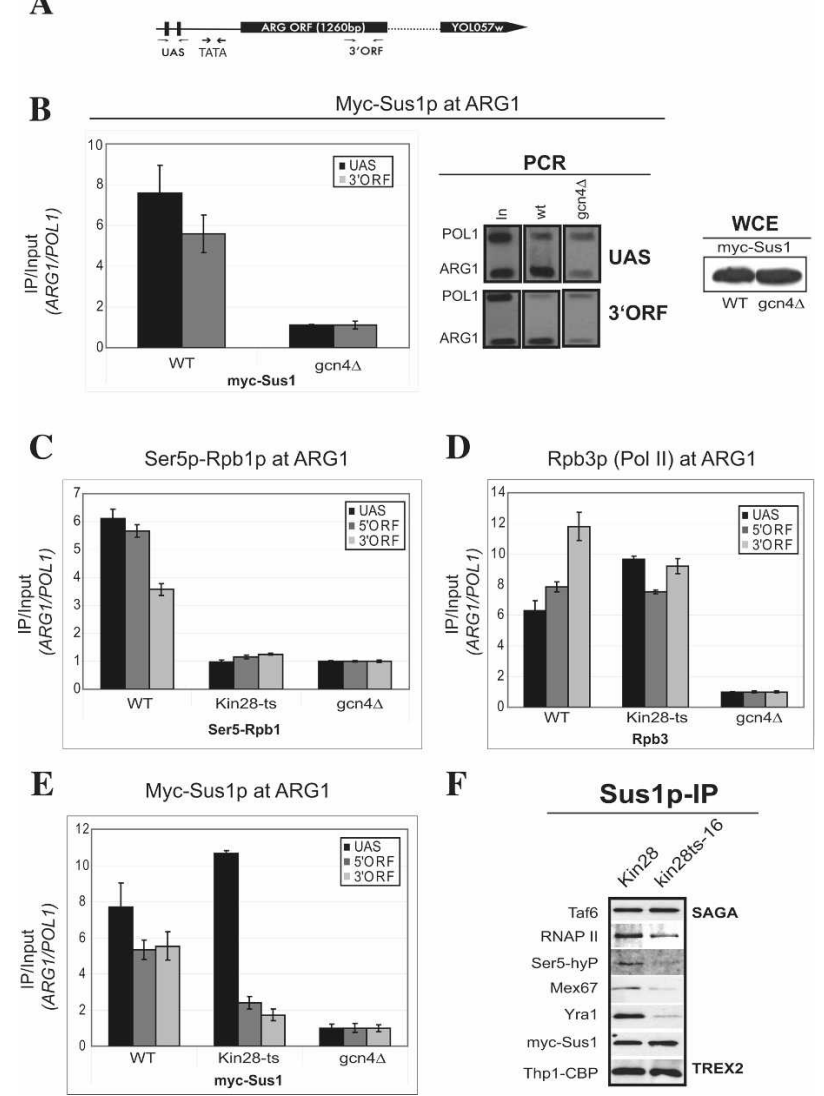

D

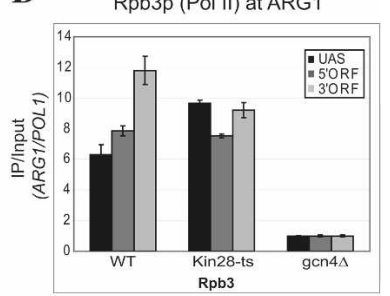

F

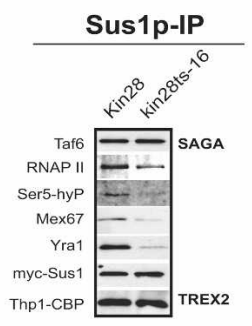

Figure 3. Sus 1 is targeted to the ARG1 coding sequence, dependent on CTD phosphorylation by Kin28. (A) ARG1 showing primers for ChIP analysis and their locations relative to the beginning of the ORF. (B) Sus1-myc GCN4 (YSR332) and Sus1myc gcn $4 \Delta$ (YSR344) cells growing at $30^{\circ} \mathrm{C}$ in SC medium lacking Ile and Val were treated with $0.6 \mathrm{mM}$ SM for $30 \mathrm{~min}$ and subjected to ChIP analysis with anti-Myc antibodies and the $A R G 1$ primers shown in $A$. The ratio of IP to input signals for Myc-Sus1p occupancy was calculated for $A R G 1$ and divided by the corresponding ratio for the reference sequence (POL1 ORF), and the mean and standard errors of the resulting normalized $\mathrm{IP} /$ input ratios from replicate cultures, calculated with three PCR measurements, are plotted. Sus1 expression in Sus1-myc GCN4 and Sus1-myc gcn4 4 was ensured by detecting myc-Sus1 from inputs by immunoblot. Representative PCR data for MycSus1p in wild type and $g c n 4 \Delta$ and Myc-Ada2 occupancy at $A R G 1$ is shown. $(C, D)$ ChIP analysis was performed as in $B$ after transferring the culture for $30 \mathrm{~min}$ to $37^{\circ} \mathrm{C}$, and treating with SM for another $30 \mathrm{~min}$ at $37^{\circ} \mathrm{C}$ to measure occupancy of RpblpCTD phosphorylated on Ser5 (S5p-Rpblp) and Rpb3p at ARG1 in a Kin28-ts16 mutant. (E) Sus12-myc KIN28-HA (YSR336), Sus1-myc kin28-HA-ts16 (YSR334), and Sus1-myc gcn4s cells were cultured and treated as in $D$ and $E$ and then subjected to ChIP analysis as in B-E. All error bars represent the SEM. $(F)$ Cells (Sus1-myc KIN28-HA [Kin28] and Sus1-myc kin28-HAts16 [kin28-HA-ts16]) growing exponentially were lysated after treatment at $37^{\circ} \mathrm{C}$, and Myc-tagged Sus1 was inmunoprecipitated from extracts. Bound proteins to Sus1-myc in each strain were analyzed with the indicated antibodies.

form of RNAP II, and that this modification stimulates the association of mRNA processing factors (Sims et al.

2004), we asked whether Kin28, the kinase responsible for this modification, affects Sus 1 recruitment to the ARG1 ORF. Wild-type and kin28-ts16 mutant cells growing at $25^{\circ} \mathrm{C}$ were shifted for $30 \mathrm{~min}$ to $37^{\circ} \mathrm{C}$ and treated with SM for another $30 \mathrm{~min}$ at $37^{\circ} \mathrm{C}$. To ensure that Kin28 was inactivated but RNAP II occupancy was not reduced, we checked the occupancies of S5-hyP RNAP II and Rpb3 (total RNAP II) at ARG1 by ChIP. As expected (Govind et al. 2007), the amount of S5-hyP RNAP II relative to $R p b 3$ was higher at the promoter than at the $5^{\prime}$ and $3^{\prime}$ ends of ARG1 in wild-type cells, and the occupancy of S5-hyP but not Rbp3 was reduced by kin28-ts16 (Fig. 3C,D). Importantly, the presence of Sus1 at the $5^{\prime}$ and $3^{\prime}$ ARG1 coding sequences, but not at the UAS, is clearly reduced after inactivation of Kin28p (Fig. 3E). Therefore, we conclude that Ser5CTD hyperphosphorylation promotes Sus1 recruitment to the ARG1 ORF without affecting its presence at the promoters.

Stimulation of Sus1 recruitment to coding regions by Kin28 dependent on RNAP II phosphorylation suggests that Ser5-P could be necessary for Sus1 association to chromatin. To study Sus1 global association with chromatin, we performed a chromatin-binding assay wherein a chromatin pellet $(\mathrm{P})$ and soluble fraction $(\mathrm{S})$ are prepared and probed by Western blot analysis. We observed an obvious shift in the distribution of Sus1 to the soluble pool in kin28-ts16 cells (Supplemental Fig. 2B), indicating that phosphorylation of RNAP II Ser5 is required for overall Sus1 association with chromatin. Moreover, as expected from the ChIP results, inactivation of Kin28 strongly reduced Sus1 copurification with RNAP II, Yra1, and Mex67, but not with SAGA (Taf6) or TREX2 (Thp1) (Fig. 3F). These results led to the idea that Sus1 contacts the export factors in the coding sequences during transcription elongation. Accordingly, we found that Mex67 is recruited to the ARG1 ORF, starting from the $5^{\prime}$ ORF to the 3' ORF (Supplemental Fig. 2C), following a pattern similar to published observations for elongation factors Spt4 and Paf1 complex (Qiu et al. 2006). Strikingly, in sus $1 \Delta$ cells, Mex67-GFP is localized to the entire nuclear rim as in wild type; however, a single strong focus several times the intensity of the rest of the nuclear rim is visible in some cells (Supplemental Fig. 2D), similar to the phenotype exhibited by Sac3 mutants (Fig. 5A in Lei et al. 2003). Thus, we conclude that Sus1 requires Ser5-P to associate with downstream factors likely coupling different steps during mRNA biogenesis.

\section{Sus1 recruitment to coding regions depends on SAGA and TREX2}

Sus1 interacts with bulk chromatin and stably associates with the SAGA and TREX2 complexes. To determine the contribution of each complex to Sus1 association with chromatin, we compared by ChIP analysis the SAGA-dependent and TREX2-dependent recruitment of Sus1 to the ARG1 ORF. To analyze SAGA dependency we used the ubp8 8 strain in which Sus1-SAGA association is disrupted, but Sus1-TREX2 copurification is not affected (Kohler et al. 2006). As previously shown for the 
GAL1 UAS, we found that Ubp8 is necessary for Sus1 high-level occupancy at the ARG1 UAS, and, interestingly, ubp8s provokes a partial reduction in Sus1 binding to coding sequences (Fig. 4A). Demonstrating that SAGA is also necessary for Sus1 binding to bulk chromatin, we observed a clear enrichment in the Sus1soluble pool in a chromatin-binding assay when Ubp8 (Fig. 4B) or other SAGA members are absent (Supplemental Fig. 3A). Hence, Ubp8 and SAGA are required to efficiently target Sus1 to transcribed coding regions and bulk chromatin.

In order to address the TREX2 contribution to Sus1 association with ORFs, we analyzed Sus1 association with chromatin in a $\operatorname{sac} 3 \Delta$ mutant. Sac3 is a nucleoporin required for mRNA export and is a subunit of TREX2 (Fischer et al. 2002, 2004; Lei et al. 2003). The sac3s deletion confers a transcription defect and hyper-recombination phenotype identical to thp1s /Gallardo et al. 2003), but RNAP II ORF occupancy at the ARG1 gene is not affected (Supplemental Fig. 3B). As cited above, Sac3CID mediates Sus1 association with TREX2 (Fischer et al. 2004). To address whether Sus 1 association with bulk chromatin is affected in sac3s cells, we performed the chromatin-binding assay described above. We observed an obvious shift in the distribution of Sus1 from the chromatin bound to the soluble fraction in sac3s cells (Fig. 4C), indicating that Sac3 is required for Sus1 association with chromatin. ChIP analysis confirmed this result for the ARG1 coding sequences (Fig. 4D, gray bars), but also revealed an unexpected Sac3-dependent recruitment of Myc-Sus1 to the UAS (Fig. 4D, black bars). Given that SAGA mediates Sus1 binding to UASs (Fig. 4A; Kohler et al. 2006), it was possible that the Sac3-dependent Sus1-UAS recruitment we observed is an indirect effect of Sac3 elimination on Sus1-SAGA association. To exclude this possibility, we immunoprecipitated Myc-Sus1 from sac3s and wild-type cells and tested by Western blotting for the presence of TREX2 and several SAGA subunits. As anticipated, Sac3 deletion prevents Sus1-TREX2 association (Fig. 4E, left panel). In contrast, SAGA subunits Gen5, Taf12, Ada2, and Taf 6 were present at wild-type levels in the immune complexes from sac3s cells, indicating an intact Sus1SAGA association (Fig. 4E, right panel). Thus, we conclude that Sus1 binding to chromatin, including both UAS and ORF sequences at $A R G 1$, is partially mediated by the TREX2 member Sac3.

Sgf73 is required for Sus1 association with SAGA and TREX2 and mediates its presence at coding regions

Our findings support a model in which Sus1 recruitment to transcribing regions depends on the coordination between SAGA and TREX2. To gain insight into the mechanism that controls this process, we sought to identify proteins besides Ubp8 and Sgf11 that influence Sus1's interaction with these complexes. Therefore, we performed Sus1-TAP purification from several SAGA mutants. Although Sus1 purifications were similar to wild type for $g c n 5 \Delta$ or $s g f 29 \Delta$ extract (data not shown),
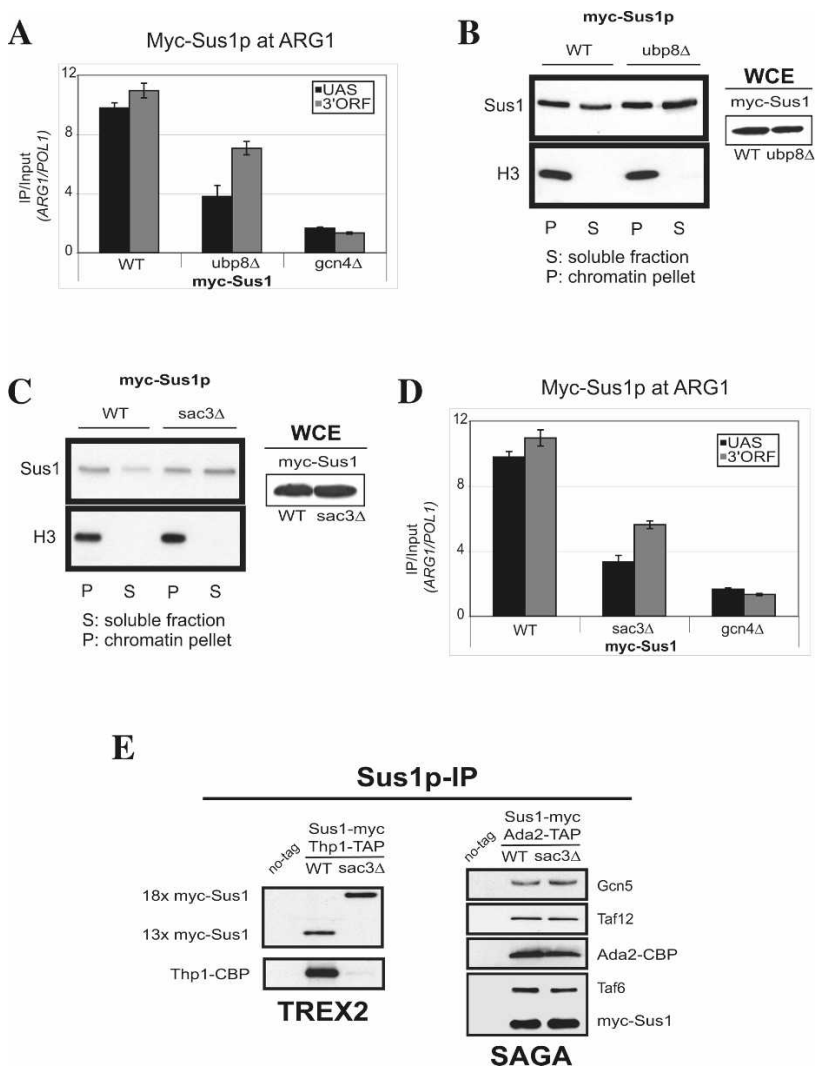

Figure 4. Sus1 requires SAGA and TREX2 to associate with chromatin. (A) ChIP analysis with anti-Myc antibodies of Sus1myc GCN4 (YSR353), Sus1-myc ubp8s (YSR188), and Sus1myc $g c n 4 \Delta$ was done as in Figure 3 . The ratio of IP to input signals for Myc-Sus1p occupancy was calculated, and the mean and standard errors of the resulting normalized IP/Input ratios from replicate cultures, calculated with three PCR measurements, are plotted. Sus1 expression in wild type and $u b p 8 \Delta$ was ensured by detecting myc-Sus 1 from inputs by Western blot. $(B)$ Wild type (BY4741) and isogenic ubp8s containing a Sus1-myc tag were grown to exponential phase, and cell lysates were subjected to a chromatin-binding assay. A chromatin pellet $(\mathrm{P})$ and a soluble fraction $(\mathrm{S})$ were obtained. Detection of Sus1 by immunoblotting using anti-myc antibody is shown. Enrichment of chromatin-associated proteins in the $\mathrm{P}$ fraction was ensured by detection of histone $\mathrm{H} 3$ by Western blot. Sus1 stability in wild type and ubp $8 \Delta$ was ensured by detecting myc-Sus 1 from whole-cell extract using the appropriate antibodies $(C)$ Wild type (BY4741) and isogenic sac3s containing Sus1-myc tag were were subjected to a chromatin-binding assay as described in $B$. $(D)$ ChIP analysis with anti-Myc antibodies of Sus1-myc GCN4, Sus1-myc sac3s (YSR373), and Sus1-myc gcn $4 \Delta$ was done as in $A$. The ratios of IP to input signals for myc-Suslp occupancy was calculated as for $A$, and resulting normalized IP/Input ratios are plotted. (E) Cells (untagged, Sus1-myc [wild type] and Sus1myc sac3s) growing exponentially were lysated, and Myctagged Sus1 was inmunoprecipitated from extracts. Inputs from the lysates and bound proteins to Sus1-myc in each strain were analyzed with the indicated antibodies.

$s g f 73 \Delta$ provoked a drastic change in the Sus1-TAP purification pattern. (Fig. 5A, lanes 1,2). Analysis by mass spectrometry revealed no copurification of any SAGA component in this mutant except for Ubp8 and Sgf11 
A

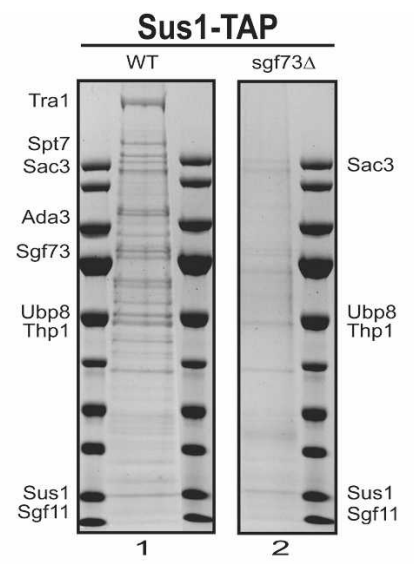

B

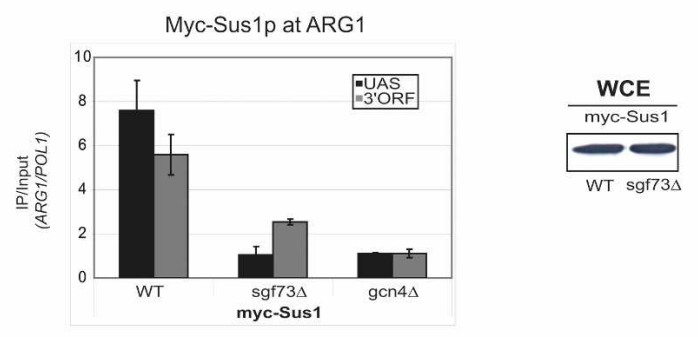

C

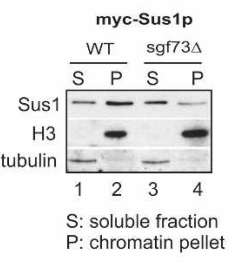

D

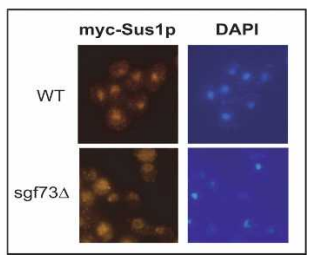

Figure 5. Association of Sus1 with SAGA, TREX2, and chromatin depends on Sgf73. (A) Sus1-TAP was purified from wild type (lane 1) or $s g f 73 \Delta$ (lane 2) by the TAP method. Purifications were analyzed on a SDS $4 \%-12 \%$ gradient polyacrylamide gel and proteins stained with Colloidal Blue are shown. Some known SAGA components are indicated as landmarks after identification by mass spectrometry. (B) ChIP analysis with anti-Myc antibodies of Sus1-myc GCN4, Sus1-myc sgf73s (YSR307), and Sus1-myc gcn4s was done as in Figure 3. The ratio of IP to input signals for Myc-Sus 1p occupancy was calculated for $A R G 1$ and divided by the corresponding ratio for the reference sequence (POL1 ORF), and the mean and standard errors of the resulting normalized IP/Input ratios from replicate cultures, calculated with three PCR measurements, are plotted. Sus 1 expression in wild type and $s g f 73 \Delta$ was ensured by detecting myc-Sus1 from inputs by Western blot. $(C)$ Wild type and $\operatorname{sgf} 73 \Delta$ containing a Sus1-myc tag were grown to exponential phase, and cell lysates were subjected to protein-chromatinenriched purification as in Figure 4B. Detection of Sus1 by Western blot using anti-myc antibody is shown. Enrichment of chromatin-associated proteins in $\mathrm{P}$ and of soluble proteins in $\mathrm{S}$ was ensured by detection of histone $\mathrm{H} 3$ and tubulin by Western blot with the appropriated antibodies. (D) DAPI-stained DNA and subcellular inmunolocalization of Sus1-myc in the indicated wild type and $s g f 73 \Delta$. In situ immunofluorescence microscopic photographs of representative cells are shown.

(data not shown). Therefore, as reported previously for ubp8s and sgf11s (Kohler et al. 2006), elimination of Sgf73 also impairs Sus1 association with SAGA. These data are consistent with a very recent report in human cells, where Sca7 (the human ortholog of Sgf73) is required for binding of the Sus1-Ubp8-Sgf11 submodule to SAGA (Zhao et al. 2008). Remarkably, in contrast to the absence of Ubp8, which does not impede Sus1 binding to TREX2, deletion of Sgf73 decreases Coomassie-stainable levels of Sac3 and Thp1 in a Sus1-TAP purification (Fig. $5 \mathrm{~A}$, lane 2). Thus, we conclude that Sgf73 plays an important role in Sus1 binding to TREX2 as well as SAGA.

We showed above that Sus1 ORF occupancy depends on SAGA (Ubp8-dependent) and TREX2 (Sac3-dependent) (Fig. 4A-D). The finding that sgf73 impairs Sus1 association with both complexes prompted us to test whether eliminating Sgf73 would evoke an even stronger decrease in Sus1 ORF occupancy. Indeed, ChIP analysis of Myc-Sus1 indicates that Sgf73 is absolutely required for Gcn4-dependent Sus1 recruitment to the ARG1 UAS (Fig. 5B, black bars), in accordance with Sgf73's role in SAGA binding to promoters (McMahon et al. 2005; Shukla et al. 2006). We found that Sgf73 is also crucial for Sus 1 targeting to coding sequences (Fig. 5B, gray bars), and, importantly, the low level of Sus1 binding to the $A R G 1$ ORF in sgf73s cells is closer to the basal level seen in $g c n 4 \Delta$ cells than that measured above in ubp $8 \Delta$ or sac3s cells (Fig. 4A,D). This supports the idea that disrupting the association of Sus1 with SAGA and TREX2 in $s g f 73 \Delta$ cells produces a compound defect in Sus1 binding to coding regions that exceeds the reduction produced by impairing either association alone. We likewise observed a severe decrease in Sus1 association with bulk chromatin in the $s g f 73 \Delta$ mutant, indicated by the drastic redistribution of Sus1 from the chromatin pellet to the soluble fraction (Fig. 5C). Furthermore, immunofluorescence analysis of Myc-Sus1 revealed partial mislocalization to the cytoplasm, as indicated by the clear reduction in nuclear signal in sgf73 $\Delta$ cells (Fig. 5D). In conclusion, Sgf73 plays a crucial role in Sus1's association with SAGA and TREX2, its binding to bulk and ARG1specific chromatin, and its nuclear association in vivo.

\section{Interplay between SAGA and TREX2 is necessary} for Sus1 function during transcription elongation

We demonstrated that Sgf73 has a pivotal role in the association between Sus1 and coding regions (Fig. 5B). The presence of Sus1 at promoters as part of SAGA and TREX2 could be a way to facilitate contact with downstream factors after transcriptional activation. If so, then it is plausible that the Sgf73-mediated interplay between SAGA and TREX2 is decisive for Sus1's role during transcription elongation, mediating the binding of Sus 1 to transcription/export factors. To explore this possibility, we extended our analysis of Sus1 interactions with RNAP II, Ser5-hyP, Mex67, and Yral to include sgf73s cells. Sus1-TAP purification from wild-type and $s g f 73 \Delta$ cells shows that Sus1 binding to Ser5-hyP is drastically reduced in the absence of Sgf73 (Fig. 6A), suggesting that Sgf73 is necessary for Sus1 binding to the Ser5 phosphorylated CTD. Strikingly, Sus1 also does not bind to the mRNA export factors Yral and Mex67 in the sgf73s mu- 
A

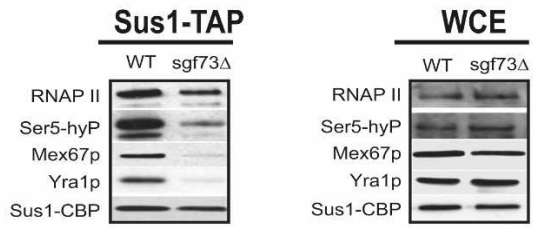

B

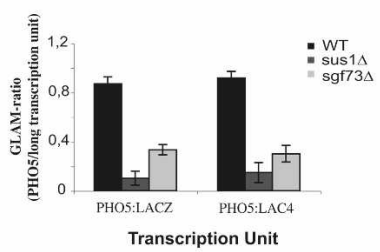

C

D

Rpb3p (Pol II) at pGAL1-YLR454w
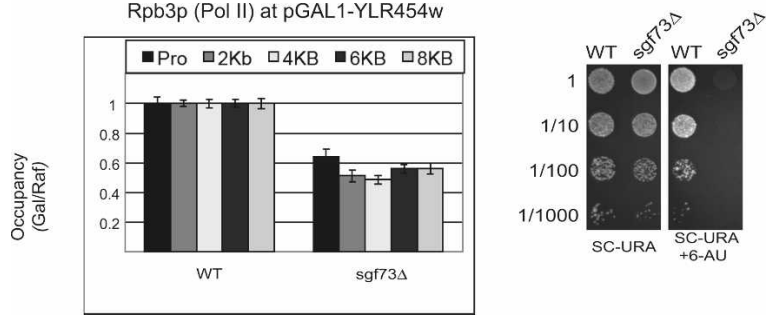

Figure 6. Sgf73 is necessary for Sus 1 copurification with RNAP II, Yra1, and Mex67 and during transcription elongation. (A) TAP purification of Sus1 TAP-tagged cells was performed from wild-type and $s g f 73 \Delta$ cells, and the eluates were resolved in $4 \%-12 \%$ SDS gradient gels, transferred, and immunoblotted. Presence of RNAP II, Ser5-hyP, Yra1, and Mex67 was revealed by the use of the appropriate antibodies as in Figure 2. Equal loading of both purifications was ensured by detecting the bait Sus1-CBP with an anti-CBP antibody. Stability of each protein in whole-cell extracts from wild-type and $s g f 73 \Delta$ backgrounds was ensured by Western blot using the mentioned antibodies. (B) Wild type (BY4741), sus1s, and sgf73s were transformed with the plasmids described in Figure 1B. GLAM-ratios are calculated as described in Figure 1C. For each strain, the average of at least three experiments was considered (error bars represent SE). (C) Comparison of Pol II (Rpb3p) occupancy at different location through the $P_{G A L 1}-Y L R 454 \mathrm{~W}$ gene following galactose induction in wild-type and $s g f 73 \Delta$ cells, expressed as the ratio of occupancies in galactose versus rafinose medium. All error bars represent the SEM. (D) 6-Azauracil (6-AU) growth assay. Wildtype (BY4741) and $s g f 73 \Delta \mathrm{URA}^{+}$strains were grown exponentially in SC-URA liquid medium, and then serial dilutions were plotted in SC-URA and SC-URA containing $50 \mu \mathrm{g} / \mathrm{mL}$ of 6 -AU. Plates were incubated for $4 \mathrm{~d}$ at $30^{\circ} \mathrm{C}$.

tant (Fig. 6A), reinforcing the observation that Sus1 requires Ser5-P to facilitate its association with Yral and Mex67 (Fig. 3F). These results indicate that Sus1 requires SAGA and TREX2 to interact with downstream factors involved in mRNA biogenesis. Given Sgf73's role in Sus1 association with SAGA, TREX2, Ser5-hyP, and mRNA export factors, one would expect that its elimination would produce a transcription elongation defect. Indeed, sgf73s confers a marked reduction in both
GLAM-ratios (Fig. 6B) and RNAP II occupancy, both at the promoter and across the $P_{G A L 1^{-}} Y L R 454 W$ gene (Fig. $6 \mathrm{C})$. In addition, $s g f 73 \Delta$ cells exhibit sensitivity to $6-\mathrm{AU}$, a common phenotype for some elongation factor $\mathrm{mu}$ tants (Fig. 6D). These findings support the model that SAGA and TREX2 both play important roles, dependent on Sgf73, in mediating Sus1 function in mRNA biogenesis during elongation.

\section{Discussion}

We described previously a mechanism by which Sus1 couples transcription activation and mRNA export, relocating the gene to the nuclear periphery after transcriptional activation (Cabal et al. 2006). Sus1 is distinctive from other factors in being present both at gene promoters and at the nuclear pore via stable physical interactions with the SAGA and TREX2 complexes, respectively. Here, we demonstrate that Sus1 is able to activate transcription and is important for transcription elongation. Work from other laboratories has suggested a role for SAGA during elongation (Desmoucelles et al. 2002; Govind et al. 2007; Wyce et al. 2007). In addition, Morillo-Huesca et al. (2006) demonstrated that some SAGA mutants ( $g c n 5 \Delta$ and $s p t 3 \Delta)$ exhibit moderate reductions in GLAM-ratios, indicating a mild defect in a genelength-dependent step of transcription, most likely elongation. We found that sus $1 \Delta$ confers a severe decrease in GLAM-ratios, which we corroborated by qRT-PCR measurements of transcript abundance. Moreover, using the long GAL-regulated YLR454W gene, we observed a progressive decay in RNAP II occupancy, suggesting reduced Pol II processivity in the absence of Susl. These results strongly suggest that Sus1 is important for efficient transcription elongation.

Several factors involved in elongation have been shown to contact the transcription machinery directly. In this study, we show for the first time that Sus1 physically interacts with active elongating forms of RNAP II phosphorylated on Ser5 and Ser2 of the CTD. Both modified forms are highly enriched in a Sus1-TAP purification when compared with another SAGA subunit (Ada2) or a TREX2 component (Thp1), indicating that Sus1 association with elongating Pol II could occur when Sus1 establishes physical contact with factors participating in mRNA biogenesis. Consistent with a role in coupling transcription and export, we also showed that Sus1 physically interacts with the mRNA export factors Yral and Mex67, which are believed to contact the transcription machinery and be present at the coding regions of different genes during transcription elongation (Zenklusen et al. 2002; Dieppois et al. 2006). In accordance with our biochemical data, we demonstrate here that Sus1 is present in coding sequences during transcriptional activation (Fig. 3B) and that this association is stimulated by Kin28 phosphorylation of the RNAP II-CTD on Ser5. Along these lines, we also observed a decrease in Sus1 association to RNAP II and export factors when Kin28 is inactivated (Fig. 3F). A plausible mechanism is that Sus1 enters the transcribed region from the UAS, where it was 
recruited in association with SAGA. There it interacts with Ser5-hyP RNAP II and the mRNA export factors Yral and Mex67 to help ensure efficient elongation and couple mRNA synthesis to export. Supporting this model, we found that Mex67 associates with the ARG1 coding region and that sus $1 \Delta$ produces a partial mislocalization of Mex67 from the nuclear pore complex in some cells (Supplemental Fig. 2C). This phenotype is similar to the one reported previously for sac3 mutants (Lei et al. 2003), indicating a partial dependence on TREX2 for Mex67 subcellular location. Notably, neither SAGA subunit Ada2 nor Ubp8 strongly copurified with Yra1 or Mex67. Hence, Sus1 likely accomplishes a more direct role in mRNA biogenesis during transcription elongation, whereas other components of SAGA participate less directly, at the level of histone modifications. In fact, recent evidence indicates that Gen5 mediates H3 acetylation and nucleosome eviction in coding regions during transcriptional activation (Govind et al. 2007), and $\mathrm{H} 2 \mathrm{~B}$ deubiquitinylation by Ubp 8 triggers appropriate transcription elongation (Wyce et al. 2007).

To address which factors are involved in Sus1's recruitment to coding regions, we studied the contribution of SAGA and TREX2 subunits in this process. In a previous report, we demonstrated that Sus1 needs Ubp8 to bind to SAGA and to be targeted to the GAL1 UAS during activation (Kohler et al. 2006). In this study, we also investigated the role of Ubp8, and, thereby, of SAGA, in the association of Sus1 with coding sequences. We show in Figure $4 \mathrm{~A}$ that $u b p 8 \Delta$ significantly reduces Sus1 occupancy of both the UAS and ORF at ARG1. The fact that Sus1 occupancy is reduced but not eliminated in $u b p 8 \Delta$ cells strongly suggests that other factors besides SAGA target Sus1 to chromatin. Nevertheless, we cannot exclude the possibility that a minor pool of Sus 1 interacts with SAGA in $u b p 8 \Delta$. Interestingly, in this direction, we observed that Sus1 still copurifies with Sgf11 and histones in a ubp8s strain where Sus1-SAGA contact is largely impaired (Kohler et al. 2006). Thus, our findings propose the existence of an intricate pathway that mediates Sus1-chromatin interaction.

SAGA and TREX2 colocalize transiently at the nuclear periphery during transcription activation to mediate gene gating, dependent on Sus1. Accordingly, it is possible that TREX2 also contributes to overall Sus1-chromatin association. Supporting this hypothesis, we found that Sus 1 binding to bulk chromatin and its recruitment to coding sequences are reduced in the sac $3 \Delta$ mutant in which Sus1-TREX2 contact is abolished (Fig. 4C-E; Fischer et al. 2004). Our ChIP results show a reduction of Sus 1 occupancy of the ARG1 ORF in sac3s cells similar in magnitude to that seen in ubp $8 \Delta$ cells. Unexpectedly, we also observed comparable reductions in Sus1 binding to the UAS in sac3s and ubp8s cells. By coimmunoprecipitation analysis, we excluded the possibility that the reduced UAS occupancy of Sus1 in sac3s cells was an indirect effect of disrupting Sus1-SAGA contact. Interestingly, we also observed a Sac3-dependent Sus1 association with the $A D H 1$ coding region (data not shown). Thus, we provide the first direct evidence that TREX2 influences Sus1's role in transcription, at least partly by promoting its binding to chromatin.

Our findings suggest a model in which SAGA and TREX2 act in concert to facilitate Sus1 function in coding regions during transcription elongation. By searching for mutants that affect Sus1 interaction with both complexes, we discovered that $s g f 73 \Delta$ drastically changes the Sus1-TAP purification profile (Fig. 5A). Similar to $u b p 8 \Delta$, Sus1-SAGA interaction is disrupted by $s g f 73 \Delta$. Interestingly, $s g f 73 \Delta$ differs from $u b p 8 \Delta$ in also decreasing the copurification of Sus1 with Sac3 and Thp1, indicating disruption of the Sus1-TREX2 interaction. Consistent with the purification data, sgf73s conferred a larger reduction in Sus1 occupancy of $A R G 1$ UAS and 3' ORF sequences (Fig. 5B) than occurred in ubp $8 \Delta$ or sac $3 \Delta$ cells, where Sus1 binding to SAGA or TREX2 alone is impaired. Consistently, we also saw a marked decrease in Sus1 binding to bulk chromatin, and partial mislocalization of Sus1p to the cytoplasm, in the absence of Sgf73. These findings indicate that abolishing Sus1 ORF occupancy by $s g f 73 \Delta$ should impede interaction with downstream factors and reduce the efficiency of elongation. This prediction was borne out by our findings that sgf73s disrupts copurification of Sus1 with RNAP II Ser5-hyP and the export factors Yral and Mex67 (Fig. 6A). Importantly, sgf73 $\Delta$ cells also display a strong reduction in GLAM-ratios, RNAP II occupancy of the $P_{\text {GAL1 }}$-YLR454w ORF, and 6AU sensitivity (Fig. 6B-D). Our data support the idea that Sus1 function during elongation is dependent on its physical connections via Sgf73 to SAGA and TREX2. Nevertheless, the fact that in sgf73 $\Delta$ a residual pool of Sus1 bound to the ARG1 3' end is detected indicates that the reduced amount of TREX2 that copurifies with Sus1 could still partially target it to ORFs. However, we cannot exclude the possibility of a SAGA-independent mechanism that targets Sus1 to transcribed regions.

In light of our findings, we propose that both SAGA and TREX2 are necessary for efficient Sus1 recruitment to chromatin and for its function in transcription coupled to mRNA export. After transcription activation (Fig. 7, top panel), Sus1 occupancy at the UAS detected by ChIP could be the combination of Sus1 bound to SAGA recruited to the promoter and Sus1 bound to TREX2 in proximity to the promoter when localized to the NPC. Once there, Sus1 can enter the coding region stimulated by RNAP II CTD-Ser5 phosphorylation and contact mRNA export factors during transcription elongation (illustrated by the orange arrow in Fig. 7). Deletion of factors that impair only the binding of Sus1 to SAGA $(u b p 8 \Delta)$ or to TREX2 $(\operatorname{sac} 3 \Delta)$ provokes a partial reduction in Sus1 UAS and 3' ORF occupancy, whereas $s g f 73 \Delta$, which affects both complexes, produces a more drastic effect in Sus1 binding throughout the gene (represented by different block arrows in Fig. 7, bottom panels). The absence of Sgf73 also impairs Sus1 binding to other factors during elongation, and, consistently, sgf73 $\Delta$ exhibits transcription elongation defects. We speculate that Sgf73 could be one of the SAGA subunits that mediate SAGA binding to TREX2. One possibility is that 


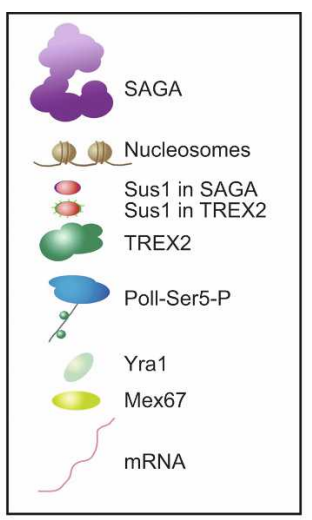

WILD-TYPE

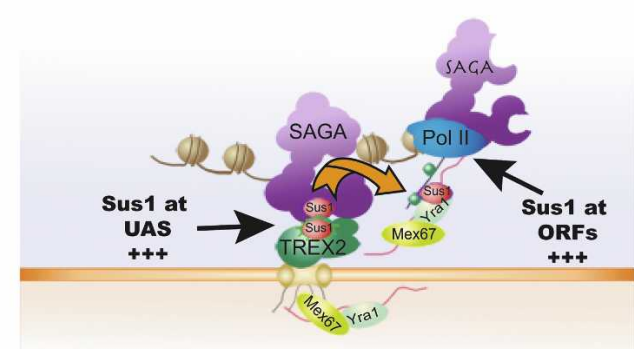

TRANSCRIPTION ON

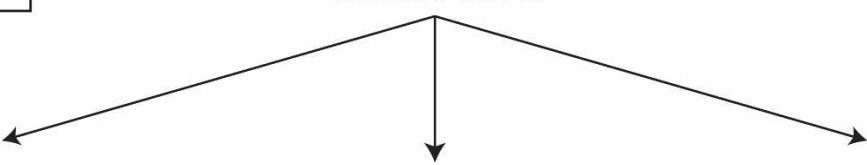

ubp8s

$\operatorname{sac} 3 \Delta$

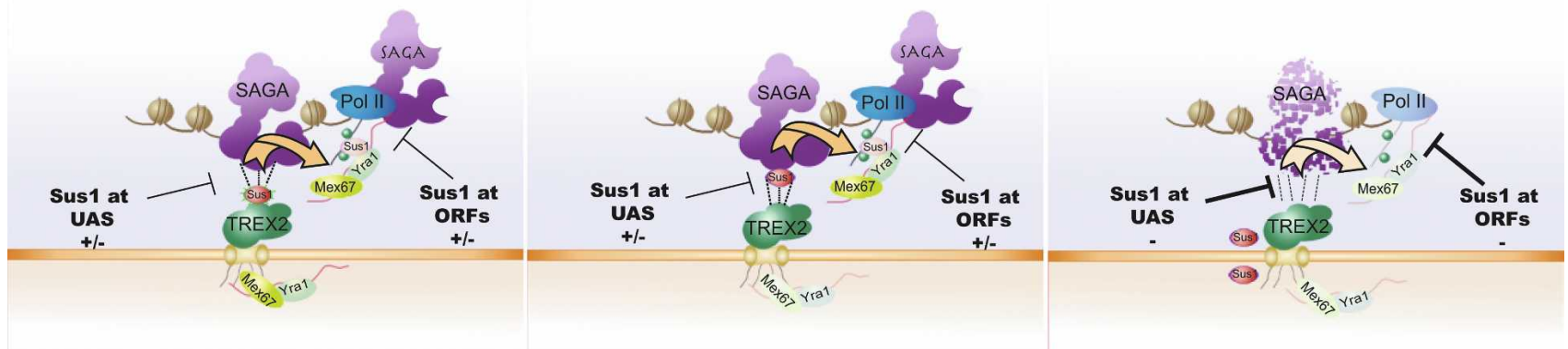

Figure 7. Coordination between SAGA and TREX2 is crucial for Sus1 function during transcription elongation. Sus1 is part of the SAGA and TREX2 complexes. Under transcription activation, SAGA is recruited to the promoter, and Sus1 enters the coding region stimulated by RNAP II CTD-Ser5 phosphorylation and contacts Yral and Mex67 during mRNA biogenesis (top panel). We observed that deletion of factors that impair only the binding of Sus1 to SAGA ( $u b p 8 \Delta$ ) or to TREX2 (sac3s) provokes a partial reduction in Sus1 UAS and 3' ORF occupancy. In sgf73s cells, in which Sus1 interaction with both complexes is affected, a more drastic effect in Sus1 binding along the transcribing region and association with export factors is observed (bottom panels).

Sgf73 alters Sus1's structure or function in a manner that prevents its association with both TREX2 and SAGA. Mislocalization of Sus1 in $s g f 73 \Delta$ cells would impede correct TREX2 formation and, consequently, SAGATREX2 contact. Interestingly, while revising this paper, the Hurt laboratory (Kohler et al. 2008) published that Sgf73 is required for $\mathrm{H} 2 \mathrm{~B}$ deubiquitinylation and that it mediates stable association between Sac3-Thp1 and Sus1-Cdc31, which strongly supports our findings. Future work on Sgf73's function will help us to clarify the nature of the SAGA-TREX2 interaction.

In summary, we demonstrate that Sus1 plays a critical role in the coordination of gene transcription and mRNA export through physical contacts with coding regions and factors involved in transcription and mRNA export. These findings support a mechanism in which SAGA and TREX2 facilitate the recruitment and function of Sus1 in coding regions during transcription elongation to coordinate mRNA biogenesis. Since Sus1 is conserved from yeast to humans, it is possible that our findings will aid understanding of how SAGA and TREX2 are interconnected in higher eukaryotic cells, where defects in Sgf73 (Sca7) and Ubp8 (USP22) are involved in neurode- generation and cancer, respectively (Helmlinger et al. 2004a,b; McMahon et al. 2005; Zhang et al. 2008).

\section{Materials and methods}

Yeast strains, DNA recombinant work, and microbiological techniques

See Supplemental Material for a detailed description. Yeast strains used in this study are listed in Supplemental Table S1 and described in the Supplemental Material, along with the antibodies and PCR primers employed (Supplemental Table S2). Microbiological techniques and yeast plasmid transformation were done essentially as described previously (Rodríguez-Navarro et al. 2004). Chromosomal integration of TAP (TRP1 or URA3 marker) and MYC (HIS3 marker) as C-terminal tags was performed as described previously (Longtine et al. 1998; Gavin et al. 2002). Strains were grown under standard conditions. For spotting analyses, cells were growth to 0.5 OD600 and subjected to 10 -fold serial dilutions. Sensitivity to $6-\mathrm{AU}(50 \mu \mathrm{g} / \mathrm{mL})$ was assayed on minimal media lacking uracil; when needed, ura3 $\Delta$ cells were transformed with a CEN/ARS URA3 plasmid.

$\beta$-Galactosidase and GLAM assays

Cells transformed with the appropriate plasmids were grown to mid-log phase in selective synthetic medium lacking trypto- 
phan with $2 \%$ glucose for the $\beta$-galactosidase assay or lacking uracil with $2 \%$ galactose for the GLAM assay. $\beta$ - Galactosidase activity was assayed in permeabilized cells and expressed in Miller units as described elsewhere. Acid phosphatase activity of the GLAM assaywas assayed as described (Morillo-Huesca et al. 2006).

TAP purifications, immunoprecipitations, and Western blot analysis

Purification of Sus1-TAP, Ada2-TAP, Ubp8-TAP, and Thp1TAP in wild-type and mutant strains was performed as described previously (Rodríguez-Navarro et al. 2004) and in the Supplemental Material. Sus1-MYC immunoprecipitation in wild type and mutants was performed as follows: $50 \mathrm{~mL}$ of cells expressing Sus1-MYC were grown on rich medium to a 0.5 OD600. Cells were harvested, washed with water, and resuspended in $250 \mu \mathrm{L}$ of lysis buffer $(50 \mathrm{mM}$ HEPES-KOH at $\mathrm{pH} 7.5$, $140 \mathrm{mM} \mathrm{NaCl}, 1 \mathrm{mM}$ EDTA, 10\% glycerol, 0.5\% NP-40, $1 \mathrm{mM}$ PMSF, protease inhibitors). An equal volume of glass beads was added. Breakage was achieved by four pulses of vortexing during $1 \mathrm{~min}$ at $4^{\circ} \mathrm{C}$. The extracts clarified were immunoprecipitated during $1 \mathrm{~h}$ at $4^{\circ} \mathrm{C}$ using anti-Myc antibody (9E10). The immunoprecipitates were washed three times for $10 \mathrm{~min}$ with $1 \mathrm{~mL}$ of lysis buffer and subsequently resuspended in $50 \mu \mathrm{L}$ of SDSPAGE sample buffer. Western analysis was performed using appropriate antibodies described in the Supplemental Material. Whole-cell extracts were conducted by trichloroacetic acid extraction. Soluble $(\mathrm{S})$ and chromatin $(\mathrm{P})$ fractions were prepared from whole-cell extracts after spheroplast lysis as described (Uhlmann et al. 1999).

\section{ChIP}

ChIPs were performed as described previously (Govind et al. 2007). After elution, $2 \mu \mathrm{L}$ of resuspended DNA were used for each PCR reaction, employing the appropriate primers listed in Supplemental Table S2.

\section{Miscellaneous}

In situ immunofluorescence of Sus1-MYC and localization of Sus1-GFP, Mex67-GFP in wild type, and sus1 $1 \Delta$ were performed as described previously, using a Leica DM6000B fluorescence microscope (Leica) with a $63 \times$ PL APO objective. RT-PCR was conducted as described previously (Rodríguez-Navarro et al. 2002).

\section{Acknowledgments}

We are grateful to Dr. J. Workman, Dr C. Dargemont, and Dr. P. Sanz for yeast strains, antibodies, or plasmids. We thank Dr. Jimeno for her contribution. The excellent technical assistance of the Mass Spectrometry service in the CIPF (ProteoRed Network), especially Dr. Valero, is acknowledged. S.C. is supported by MEC (BFU2007-67575-C03-02) and the Andalusian Government (CVI-271). This work was supported partly by the Intramural Research Program of the NIH. S.R.-N. is supported by the Ramón y Cajal program and funded by the MEC (BFU200506856) and the GV (ACOMP06/165 and ACOMP07/013). P.P.G. and B.C.-B. are holders of predoctoral fellowships from the CIPF and MEC, respectivelly.

\section{References}

Abruzzi, K.C., Lacadie, S., and Rosbash, M. 2004. Biochemical analysis of TREX complex recruitment to intronless and intron-containing yeast genes. EMBO J. 23: 2620-2631.
Baker, S.P. and Grant, P.A. 2007. The SAGA continues: Expanding the cellular role of a transcriptional co-activator complex. Oncogene 26: 5329-5340.

Bhaumik, S.R. and Green, M.R. 2001. SAGA is an essential in vivo target of the yeast acidic activator Gal4p. Genes \& Dev. 15: 1935-1945.

Cabal, G.G., Genovesio, A., Rodríguez-Navarro, S., Zimmer, C., Gadal, O., Lesne, A., Buc, H., Feuerbach-Fournier, F., OlivoMarin, J.C., Hurt, E.C., et al. 2006. SAGA interacting factors confine sub-diffusion of transcribed genes to the nuclear envelope. Nature 441: 770-773.

Candau, R., Zhou, J.X., Allis, C.D., and Berger, S.L. 1997. Histone acetyltransferase activity and interaction with ADA2 are critical for GCN5 function in vivo. EMBO J. 16: 555-565.

Chavez, S., Beilharz, T., Rondon, A.G., Erdjument-Bromage, H., Tempst, P., Svejstrup, J.Q., Lithgow, T., and Aguilera, A. 2000. A protein complex containing Tho2, Hpr1, Mftl and a novel protein, Thp2, connects transcription elongation with mitotic recombination in Saccharomyces cerevisiae. EMBO J. 19: 5824-5834.

Chekanova, J.A., Abruzzi, K.C., Rosbash, M., and Belostotsky, D.A. 2008. Sus1, Sac3, and Thp1 mediate post-transcriptional tethering of active genes to the nuclear rim as well as to non nascent mRNP. RNA 14: 66-77.

Cole, C.N. and Scarcelli, J.J. 2006. Transport of messenger RNA from the nucleus to the cytoplasm. Curr. Opin. Cell Biol. 18: 299-306.

Cosma, M.P., Tanaka, T., and Nasmyth, K. 1999. Ordered recruitment of transcription and chromatin remodeling factors to a cell cycle- and developmentally regulated promoter. Cell 97: 299-311.

Desmoucelles, C., Pinson, B., Saint-Marc, C., and DaignanFornier, B. 2002. Screening the yeast 'disruptome' for mutants affecting resistance to the immunosuppressive drug, mycophenolic acid. J. Biol. Chem. 277: 27036-27044.

Dieppois, G., Iglesias, N., and Stutz, F. 2006. Cotranscriptional recruitment to the mRNA export receptor Mex67p contributes to nuclear pore anchoring of activated genes. Mol. Cell. Biol. 26: 7858-7870.

Fischer, T., Strasser, K., Racz, A., Rodríguez-Navarro, S., Oppizzi, M., Ihrig, P., Lechner, J., and Hurt, E. 2002. The mRNA export machinery requires the novel Sac3p-Thplp complex to dock at the nucleoplasmic entrance of the nuclear pores. EMBO J. 21: 5843-5852.

Fischer, T., Rodríguez-Navarro, S., Pereira, G., Racz, A., Schiebel, E., and Hurt, E. 2004. Yeast centrin Cdc31 is linked to the nuclear mRNA export machinery. Nat. Cell Biol. 6: 840848.

Gallardo, M., Luna, R., Erdjument-Bromage, H., Tempst, P., and Aguilera, A. 2003. Nab2p and the Thp1p-Sac3p complex functionally interact at the interface between transcription and mRNA metabolism. J. Biol. Chem. 278: 24225-24232.

Gavin, A.C., Bosche, M., Krause, R., Grandi, P., Marzioch, M., Bauer, A., Schultz, J., Rick, J.M., Michon, A.M., Cruciat, C.M., et al. 2002. Functional organization of the yeast proteome by systematic analysis of protein complexes. Nature 415: 141-147.

Govind, C.K., Zhang, F., Qiu, H., Hofmeyer, K., and Hinnebusch, A.G. 2007. Gcn5 promotes acetylation, eviction, and methylation of nucleosomes in transcribed coding regions. Mol. Cell 25: 31-42.

Grant, P.A., Duggan, L., Cote, J., Roberts, S.M., Brownell, J.E., Candau, R., Ohba, R., Owen Hughes, T., Allis, C.D., Winston, F., et al. 1997. Yeast Gen5 functions in two multisubunit complexes to acetylate nucleosomal histones: Characterization of an Ada complex and the SAGA (Spt/Ada) com- 
plex. Genes \& Dev. 11: 1640-1650.

Helmlinger, D., Abou-Sleymane, G., Yvert, G., Rousseau, S., Weber, C., Trottier, Y., Mandel, J.L., and Devys, D. 2004a. Disease progression despite early loss of polyglutamine protein expression in SCA7 mouse model. J. Neurosci. 24: 18811887.

Helmlinger, D., Hardy, S., Sasorith, S., Klein, F., Robert, F., Weber, C., Miguet, L., Potier, N., Van-Dorsselaer, A., Wurtz, J.M., et al. 2004b. Ataxin-7 is a subunit of GCN5 histone acetyltransferase-containing complexes. Hum. Mol. Genet. 13: $1257-1265$.

Henry, K.W., Wyce, A., Lo, W.S., Duggan, L.J., Emre, N.C., Kao, C.F., Pillus, L., Shilatifard, A., Osley, M.A., and Berger, S.L. 2003. Transcriptional activation via sequential histone H2B ubiquitylation and deubiquitylation, mediated by SAGA-associated Ubp8. Genes \& Dev. 17: 2648-2663.

Ingvarsdottir, K., Krogan, N.J., Emre, N.C., Wyce, A., Thompson, N.J., Emili, A., Hughes, T.R., Greenblatt, J.F., and Berger, S.L. 2005. H2B ubiquitin protease Ubp8 and Sgf11 constitute a discrete functional module within the Saccharomyces cerevisiae SAGA complex. Mol. Cell. Biol. 25: 1162-1172.

Kohler, A. and Hurt, E. 2007. Exporting RNA from the nucleus to the cytoplasm. Nat. Rev. Mol. Cell Biol. 8: 761-773.

Kohler, A., Pascual-Garcia, P., Llopis, A., Zapater, M., Posas, F., Hurt, E., and Rodríguez-Navarro, S. 2006. The mRNA export factor Sus1 is involved in Spt/Ada/Gen5 acetyltransferasemediated $\mathrm{H} 2 \mathrm{~B}$ deubiquitinylation through its interaction with Ubp8 and Sgf11. Mol. Biol. Cell 17: 4228-4236.

Kohler, A., Schneider, M., Cabal, G.G., Nehrbass, U., and Hurt, E. 2008. Yeast Ataxin-7 links histone deubiquitination with gene gating and mRNA export. Nat. Cell Biol. 10: 707-715.

Komili, S. and Silver, P.A. 2008. Coupling and coordination in gene expression processes: A systems biology view. Nat. Rev. Genet. 9: 38-48.

Kurshakova, M., Maksimenko, O., Golovnin, A., Pulina, M., Georgieva, S., Georgiev, P., and Krasnov, A. 2007a. Evolutionarily conserved $\mathrm{E} / \mathrm{y} / 2 / \mathrm{Sus} 1$ protein is essential for the barrier activity of $\mathrm{Su}(\mathrm{Hw})$-dependent insulators in Drosophila. Mol. Cell 27: 332-338.

Kurshakova, M.M., Krasnov, A.N., Kopytova, D.V., Shidlovskii, Y.V., Nikolenko, J.V., Nabirochkina, E.N., Spehner, D., Schultz, P., Tora, L., and Georgieva, S.G. 2007b. SAGA and a novel Drosophila export complex anchor efficient transcription and mRNA export to NPC. EMBO J. 26: 4956-4965.

Larschan, E. and Winston, F. 2001. The S. cerevisiae SAGA complex functions in vivo as a coactivator for transcriptional activation by Gal4. Genes \& Dev. 15: 1946-1956.

Lee, K.K., Florens, L., Swanson, S.K., Washburn, M.P., and Workman, J.L. 2005. The deubiquitylation activity of Ubp8 is dependent upon Sgf11 and its association with the SAGA complex. Mol. Cell. Biol. 25: 1173-1182.

Lei, E.P., Stern, C.A., Fahrenkrog, B., Krebber, H., Moy, T.I., Aebi, U., and Silver, P.A. 2003. Sac3 is an mRNA export factor that localizes to cytoplasmic fibrils of nuclear pore complex. Mol. Biol. Cell 14: 836-847.

Longtine, M.S., McKenzie 3rd, A., Demarini, D.J., Shah, N.G., Wach, A., Brachat, A., Philippsen, P., and Pringle, J.R. 1998. Additional modules for versatile and economical PCR-based gene deletion and modification in Saccharomyces cerevisiae. Yeast 14: 953-961.

McMahon, S.J., Pray-Grant, M.G., Schieltz, D., Yates 3rd, J.R., and Grant, P.A. 2005. Polyglutamine-expanded spinocerebellar ataxia-7 protein disrupts normal SAGA and SLIK histone acetyltransferase activity. Proc. Natl. Acad. Sci. 102: 8478-8482.
Morillo-Huesca, M., Vanti, M., and Chavez, S. 2006. A simple in vivo assay for measuring the efficiency of gene length-dependent processes in yeast mRNA biogenesis. FEBS J. 273: 756769

Nagy, Z. and Tora, L. 2007. Distinct GCN5/PCAF-containing complexes function as co-activators and are involved in transcription factor and global histone acetylation. Oncogene 26: $5341-5357$.

Qiu, H., Hu, C., Wong, C.M., and Hinnebusch, A.G. 2006. The Spt4p subunit of yeast DSIF stimulates association of the Paf1 complex with elongating RNA polymerase II. Mol. Cell. Biol. 26: 3135-3148.

Rodríguez-Navarro, S., Igual, J.C., and Perez-Ortin, J.E. 2002. SRC1: An intron-containing yeast gene involved in sister chromatid segregation. Yeast 19: 43-54.

Rodríguez-Navarro, S., Fischer, T., Luo, M.J., Antunez, O., Brettschneider, S., Lechner, J., Perez Ortin, J.E., Reed, R., and Hurt, E. 2004. Sus1, a functional component of the SAGA histone acetylase complex and the nuclear pore-associated mRNA export machinery. Cell 116: 75-86.

Saunders, A., Core, L.J., and Lis, J.T. 2006. Breaking barriers to transcription elongation. Nat. Rev. Mol. Cell Biol. 7: 557567.

Shukla, A., Bajwa, P., and Bhaumik, S.R. 2006. SAGA-associated Sgf73p facilitates formation of the preinitiation complex assembly at the promoters either in a HAT-dependent or independent manner in vivo. Nucleic Acids Res. 34: 62256232.

Sims III, R.J., Belotserkovskaya, R., and Reinberg, D. 2004. Elongation by RNA polymerase II: The short and long of it. Genes \& Dev. 18: 2437-2468.

Strasser, K., Masuda, S., Mason, P., Pfannstiel, J., Oppizzi, M., Rodríguez-Navarro, S., Rondon, A.G., Aguilera, A., Struhl, K., Reed, R., et al. 2002. TREX is a conserved complex coupling transcription with messenger RNA export. Nature 417: 304-308.

Swanson, M.J., Qiu, H., Sumibcay, L., Krueger, A., Kim, S.J., Natarajan, K., Yoon, S., and Hinnebusch, A.G. 2003. A multiplicity of coactivators is required by Gen $4 p$ at individual promoters in vivo. Mol. Cell. Biol. 23: 2800-2820.

Uhlmann, F., Lottspeich, F., and Nasmyth, K. 1999. Sister-chromatid separation at anaphase Honest is promoted by cleavage of the cohesin subunit Scc1. Nature 400: 37-42.

Weake, V.M. and Workman, J.L. 2008. Histone ubiquitination: Triggering gene activity. Mol. Cell 29: 653-663.

Wyce, A., Xiao, T., Whelan, K.A., Kosman, C., Walter, W., Eick, D., Hughes, T.R., Krogan, N.J., Strahl, B.D., and Berger, S.L. 2007. H2B ubiquitylation acts as a barrier to Ctk1 nucleosomal recruitment prior to removal by Ubp 8 within a SAGArelated complex. Mol. Cell 27: 275-288.

Zenklusen, D., Vinciguerra, P., Wyss, J.C., and Stutz, F. 2002. Stable mRNP formation and export require cotranscriptional recruitment of the mRNA export factors Yralp and Sub2p by Hprlp. Mol. Cell. Biol. 22: 8241-8253.

Zhang, X.Y., Varthi, M., Sykes, S.M., Phillips, C., Warzecha, C., Zhu, W., Wyce, A., Thorne, A.W., Berger, S.L., and McMahon, S.B. 2008. The putative cancer stem cell marker USP22 is a subunit of the human SAGA complex required for activated transcription and cell-cycle progression. Mol. Cell 29: 102-111.

Zhao, Y., Lang, G., Ito, S., Bonnet, J., Metzger, E., Sawatsubashi, S., Suzuki, E., Le Guezennec, X., Stunnenberg, H.G., Krasnov, A., et al. 2008. A TFTC/STAGA module mediates histone $\mathrm{H} 2 \mathrm{~A}$ and $\mathrm{H} 2 \mathrm{~B}$ deubiquitination, coactivates nuclear receptors, and counteracts heterochromatin silencing. Mol Cell 29: 92-101. 


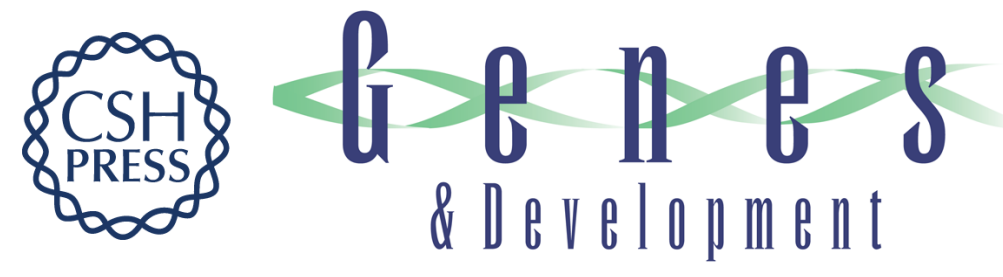

\section{Sus1 is recruited to coding regions and functions during transcription elongation in association with SAGA and TREX2}

Pau Pascual-García, Chhabi K. Govind, Ethel Queralt, et al.

Genes Dev. 2008, 22:

Access the most recent version at doi:10.1101/gad.483308

Supplemental
Material http://genesdev.cshlp.org/content/suppl/2008/10/17/22.20.2811.DC1

References This article cites 48 articles, 22 of which can be accessed free at:

http://genesdev.cshlp.org/content/22/20/2811.full.html\#ref-list-1

License

Email Alerting Receive free email alerts when new articles cite this article - sign up in the box at the top

Service

right corner of the article or click here.

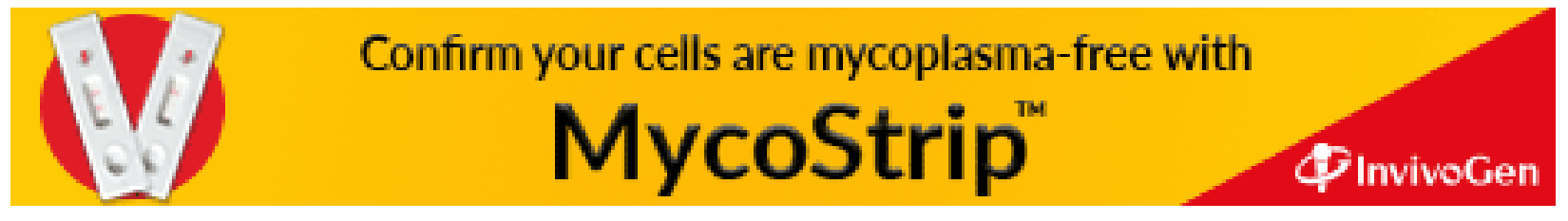

